\title{
Su Yang Decoction induces human colon carcinoma cell apoptosis by activating caspases
}

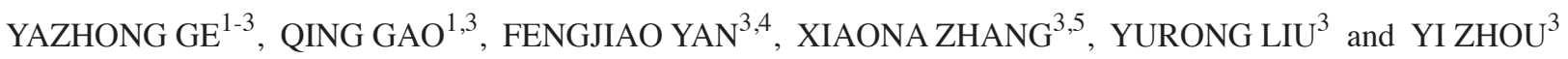 \\ ${ }^{1}$ Department of Healthy Food Development, Infinitus (China) Company Ltd., Guangzhou, Guangdong 510024; \\ ${ }^{2}$ School of Food Science and Engineering, South China University of Technology, Guangzhou, Guangdong 510102; \\ ${ }^{3}$ Department of Pharmacology, College of Pharmaceutical Science, Guangzhou Medical University, Guangzhou, \\ Guangdong 511436; ${ }^{4}$ Department of Cardiovascular Medicine, The First Affiliated Hospital of Sun-Yat-sen University, \\ Guangzhou, Guangdong 510080; ${ }^{5}$ Department of Pharmacology, The Sixth Affiliated Hospital \\ of Sun-Yat-sen University, Guangzhou, Guangdong 510520, P.R. China
}

Received October 5, 2017; Accepted July 5, 2018

DOI: $10.3892 / \mathrm{ol} .2018 .9625$

\begin{abstract}
Su Yang Decoction (SYD) is a popular healthcare product comprised of various brassicaceous vegetables known to exhibit anticancer activity. However, the effects of SYD on tumor growth, following its combination with brassicaceous vegetables into a compound formula, require further investigation. In the present study, a quality control of SYD was subjected to high-performance liquid chromatography for the quantitative and qualitative determination of sulforaphane, the main anticancer component of SYD. SYD inhibited colon cancer cell proliferation in a dose- and time-dependent manner and induced G1 phase arrest in colon cancer HT-29 cell lines. In addition, SYD triggered caspase-mediated apoptosis in a dose-dependent manner and induced the cleavage of poly (ADP-ribose) polymerase, tumor necrosis factor superfamily member 10, X-linked inhibitor of apoptosis, and truncated BH3 interacting domain death agonist. Furthermore, the expression of FADD-like interleukin-1 $\beta$-converting enzyme (FLICE)-like inhibitory proteins (FLIPs) and long isoform of FLICE-inhibitory protein was reduced by SYD and the direct targeting of cellular-FLIP with small interfering RNA inhibited colon cancer cell proliferation and decreased the SYD concentration required for proliferation inhibition. SYD treatment was also associated with the translocation of proapoptotic BCL2 associated X, apoptosis regulator to the mitochondria and the release of cytochrome $c$ from the mitochondria to the cytosol. The aforementioned results indicate that SYD exerts anti-colorectal cancer effects through an underlying mechanism that may involve caspase activation.
\end{abstract}

Correspondence to: Dr Yi Zhou, Department of Pharmacology, College of Pharmaceutical Science, Guangzhou Medical University, 1 Xiufacun Road, Guangzhou, Guangdong 511436, P.R. China E-mail: zhouyi0264@126.com

Key words: Su Yang Decoction, broccoli and green cabbage, caspase, apoptosis, human colon carcinoma

\section{Introduction}

The identification of risk factors for colon cancer has been an ongoing investigation in present research (1). With the exception of familial polyposis, the majority of risk factors have indicated controversial effects (1). However, $90 \%$ of colon cancer cases in the US and other developed countries have been reported to be attributed to environmental factors (1). Among the postulated dietary factors, vegetable and fruit consumption has been reported as the most consistent to exert an anti-carcinogenic effect $(2,3)$. It has been reported that vegetables and fruits have numerous putative anti-carcinogenic substances, providing biological support to the hypothesis that vegetable and fruit consumption may aid in the prevention of the disease (4). Su Yang Decoction (SYD) comprises two brassicaceous vegetables, broccoli and green cabbage, which have been reported to exert anticancer effects in vivo and in vitro $(5,6)$. In addition, the sulforaphane constituents in vegetables, including broccoli and green cabbage, have been reported to inhibit the proliferation of pancreatic cancer (7) and gastric cancer cells (6) and induce cancer cell apoptosis. Therefore, it has been reported that broccoli and green cabbage are considered to have anticancer properties and are extensively consumed in China (2). However, the expected therapeutic effects of SYD as a compound formula require further investigation.

Apoptosis is a form of programmed cell death that is responsible for tissue homeostasis in cancer cells and is induced by numerous cancer treatments (8). It has been indicated that apoptosis involves two major pathways: The intrinsic (mitochondrial-mediated) pathway, which involves the activation of caspase-9 (CASP9) and caspase-10, and the extrinsic [death receptor (DR)-mediated] pathway (3). In the extrinsic pathway, the binding of extracellular death ligands to their cell-surface DRs has been reported to induce caspase- 8 (CASP8) activation (4). In contrast, the intrinsic pathway has been reported to be activated by the release of proapoptotic factors, including cytochrome $c$ from the mitochondria to the cytosol and the activation of CASP9, in addition to being amplified by the CASP8-mediated cleavage of $\mathrm{BH} 3$ interacting 
domain death agonist (9). The extrinsic apoptosis pathway is initiated by the binding of death receptor ligands, including tumor necrosis factor (TNF)-related apoptosis-inducing ligand (TRAIL) or cluster of differentiation 95 ligand, to their cognate death receptors at the cell membrane (10). Active caspase- 8 activates caspase-3, resulting in apoptosis (11). As an anti-apoptotic protein, cellular FADD-like IL-1 $\beta$-converting enzyme-inhibitory protein-inhibitory protein (c-FLIP) can block death-receptor signaling by interfering with caspase- 8 activation at the DISC (10). Therefore, the present study aimed to investigate the anticancer activity of SYD on colon cancer HT-29 cells, in addition to examining the SYD anticancer underlying mechanism.

\section{Materials and methods}

Materials. High performance liquid chromatography (HPLC)-grade methanol was purchased from Sigma-Aldrich (Merck KGaA, Darmstadt, Germany). Ultrapure water was prepared using a Millipore SAS 67120 system (Merck $\mathrm{KGaA})$. The sulforaphane ( $98 \%$ purity), as the reference standard substance, was purchased from Shanghai Yuanye Biotechnology Co., Ltd., (Shanghai, China). Fetal bovine serum (FBS), penicillin G, streptomycin and amphotericin B were obtained from Gibco (Thermo Fisher Scientific, Inc., Waltham, USA). Dimethyl sulfoxide, ribonuclease (RNase), propidium iodide (PI) and RPMI-1640 were purchased from Sigma-Aldrich (Merck KGaA). Broccoli and green cabbage material were obtained from Infinitus Company Ltd. (Guangzhou, China) and were placed on dry ice and freeze-dried immediately to preserve their freshness.

HPLC-ultraviolet (UV) analysis. A Shimadzu LC-20AT HPLC system with an UV detector was used (Shimadzu Corporation, Kyoto, Japan) for quantitative determination. A Phenomenex Luna C18 column $(4.6 \times 250 \mathrm{~mm}, 5 \mu \mathrm{m}$; Guangzhou FLM Scientific Instrument Co., Ltd., Guangzhou, China) was used, according to the manufacturer's protocols, and the mobile phase composed of methanol:water $(20: 80 \% \mathrm{v} / \mathrm{v})$ at a flow rate of $1.0 \mathrm{ml} / \mathrm{min}$. Furthermore, the detection wavelength was $225 \mathrm{~nm}$ and the temperature of the column was set to $30^{\circ} \mathrm{C}$. The injection volume was $20 \mathrm{ml}$. The limit of detection was $0.2 \mu \mathrm{g} / \mathrm{ml}$. Data acquisition was performed using the LabSolutions CS software version 2.53 (Shimadzu Corporation). There are 6 points in the Standard curve (6 standard samples); additionally, a representative standard sample and tested sample were used. All experiment were repeated twice each one in duplicate.

Preparation of SYD. SYD was prepared according to the procedure described by Rose et al (7). In brief, $100 \mathrm{mg}$ of freeze-dried material, including broccoli and green cabbage at a weight ratio of 1:1, was weighed into a $50-\mathrm{ml}$ polypropylene tube and subjected to two 30 min cycles of ultrasonic disruption $(59 \mathrm{kHz})$ in $70 \%$ ethanol $(3 \mathrm{ml})$ at $70^{\circ} \mathrm{C}$. The mixture was cooled to room temperature and centrifuged at $3,000 \mathrm{x} \mathrm{g}$ for $5 \mathrm{~min}$. Following centrifugation, 1-ml aliquots were removed and condensed in a vacuum to $200 \mu \mathrm{l}$. The resulting concentrates were filtered through sterile non-pyrogenic filters $(0.2 \mu \mathrm{m}$; Merck $\mathrm{KGaA})$ and stored at $70^{\circ} \mathrm{C}$ prior to testing. The extracts for each sample yielded an equivalent concentration of $50 \mathrm{mg} / \mathrm{ml}$. The sulforaphane composition in the extracts was analyzed with a Shimadzu LC-20AT HPLC system with an UV detector (Shimadzu Corporation, Kyoto, Japan).

Cell culture. Following ethical approval by the Institutional Animal Care Committee and the Local Veterinary Office and Ethics Committee of Guangzhou Medical University (Guangzhou, China), the human colon cancer HT-29, LS-174-T and CRL-1790 cell lines were obtained from the Clinic Research Center of Guangzhou Medical University (Guangzhou, China). They were used for the subsequent proliferation, apoptosis, cell cycle and western-blotting assays. The human colon epithelial CRL-1790 cell line was obtained from American Type Culture Collection (Manassas, VA, USA). HT-29, LS-174-T and CRL-1790 cells were grown in Dulbecco's modified Eagle's medium (DMEM; Gibco; Thermo Fisher Scientific, Inc.) with 10\% FBS and $1 \%$ Penicillin G-Streptomycin and maintained at $37^{\circ} \mathrm{C}$ with $5 \% \mathrm{CO}_{2}$ atmosphere. All cell studies were approved by the Institutional Animal Care Committee and the Local Veterinary Office and Ethics Committee of Guangzhou Medical University (Guangzhou, China).

Proliferation assay. Proliferation was assessed through an MTS assay kit (Promega Corporation, Madison, WI, USA), according to the manufacturer's protocols. HT-29, LS-174-T and CRL-1790 cells were inoculated in 96-well plates at the density of $5 \times 10^{4}$ cells/well. At $0,24,48$ and $72 \mathrm{~h}$ of incubation at $37^{\circ} \mathrm{C}, 20 \mu \mathrm{l} \mathrm{MTS} / \mathrm{PMS}$ reagent was added for another $4 \mathrm{~h}$ of incubation at room temperature. Dimethyl sulfoxide was used to dissolve the purple formazan. The absorbance at $490 \mathrm{~nm}$ was detected by a microplate reader. HT-29 cells were treated by $0,10,50,100$ or $200 \mu \mathrm{g} / \mathrm{ml} \mathrm{SYD}$ for $48 \mathrm{~h}$ at $37^{\circ} \mathrm{C}$, and then lactate dehydrogenase ( $\left.\mathrm{LDH}\right)$ release was assessed with a LDH activity kit (cat. no. 03002209122; Roche Diagnostics, Indianapolis, IN, USA) and expressed as a percentage [(sample absorbance/lysed cell absorbance-control absorbance) x100]. Each individual experiment was performed in triplicate.

A total of $5 \times 10^{4}-1 \times 10^{5}$ control small interfering RNA (siRNA) or c-FLIP siRNA HT-29 cells/well were seeded in 6-well plates. After $24 \mathrm{~h}$ at $37^{\circ} \mathrm{C}$, the DMEM with $10 \% \mathrm{FBS}$ was replaced with fresh DMEM with $10 \%$ FBS and 0,100 or $200 \mu \mathrm{g} / \mathrm{ml} \mathrm{SYD}$ at $37^{\circ} \mathrm{C}$ and incubated for 7 days. Cell viability was determined by Typan Blue staining at room temperature for $30 \mathrm{sec}$ and direct cell counting using a hematocytometer (Thorlabs, Inc., Newton, NJ, USA).

Flow cytometry analysis of the cell cycle distribution. The flow cytometric analysis was performed as described previously (12). Subconfluent cultures of HT-29 cells $\left(1.0 \times 10^{5}\right.$ cells/well) were treated with either PBS or $0,100,200$ and $400 \mu \mathrm{g} / \mathrm{ml} \mathrm{SYD}$. Subsequent to 24,48 and $72 \mathrm{~h}$ of treatment at room temperature, the cells were harvested by brief trypsinization and centrifugation at room temperature at 3,000 $\mathrm{x}$ g for $5 \mathrm{~min}$. The cell pellets were washed twice with ice-cold PBS and $0.5 \times 10^{6}$ cells were suspended in $500 \mathrm{ml}$ of saponin/PI solution $[0.3 \%(\mathrm{w} / \mathrm{v})$ saponin; $25 \mu \mathrm{g} / \mathrm{ml} \mathrm{PI} ; 0.1 \mathrm{mmol} / \mathrm{L}$ EDTA and $10 \mu \mathrm{g} / \mathrm{ml}$ RNase A in PBS] and incubated at $4^{\circ} \mathrm{C}$ for $24 \mathrm{~h}$ in the 
dark. Apoptosis was quantified using an annexin V-fluorescein isothiocyanate (FITC) kit (R\&D Systems, Inc., Minneapolis, MN, USA), according to the manufacturer's protocols. The stained cells were analyzed using a FACS flow cytometer (BD Biosciences, Franklin Lakes, NJ, USA) and FCS Express V3.0 software (De Novo Software, Glendale, CA, USA). ModFit LT software v3.3 (Verity Software House, Inc., Topsham, ME, USA) was used to analyze the results of the cell cycle.

Colorimetric caspase activation assay. HT-29 cells $\left(1 \times 10^{6}\right)$ were seeded into $6 \mathrm{~cm}$ dishes and treated with $0,100,200$ and $400 \mu \mathrm{g} / \mathrm{ml} \mathrm{SYD}$ for the indicated times, the activities of caspase-3 (CASP3), CASP8 and CASP9 were measured by cleavage of a $5 \mu 1$ IETD-@NA substrate (caspase-8) or DEVD-@NA substrate (caspase-3, 9). CAS activity was measured using the Colorimetric CaspACE assay system, and the activities of CASP8 (cat. no. AAH-APO-1-2) and CASP9 (cat. no. 68FL-Casp9-S100) were determined with CASP8 and CASP9 Colorimetric assay kits, according to the manufacturer's protocols (RayBiotech Life, Norcross, GA, USA). To inhibit the tested caspases, the cells were preincubated at $37^{\circ} \mathrm{C}$ for $2 \mathrm{~h}$ with $50 \mathrm{mM}$ Z-DEVD-FMK (cat. no. A1920; CASP3 inhibitor), Z-IETD-FMK (cat. no. B3232; CASP8 inhibitor), Z-LEHD-FMK (cat. no. B3233; CASP9 inhibitor) and Z-VAD-FMK (cat. no. A1902; Pan-caspase inhibitor) (all from ApexBio, Shanghai, China).

Measurement of the release of cytochrome $c$. The release of cytochrome $c$ from the mitochondria of HT-29 cells into the cytosol was measured using a streptavidin-peroxidase immunohistochemical kit (cat. no. SP0041; OriGene Technologies, Inc., Beijing, China) as described previously (13). Briefly, following incubation at $37^{\circ} \mathrm{C}$ for $24 \mathrm{~h}$, HT-29 cells $\left(1 \times 10^{6}\right)$ were seeded into $6 \mathrm{~cm}$ dishes and were exposed to either $100 \mu \mathrm{g} / \mathrm{ml}$ or $200 \mu \mathrm{g} / \mathrm{ml} \mathrm{SYD} \mathrm{or} \mathrm{left} \mathrm{untreated} \mathrm{(control} \mathrm{group).} \mathrm{Subsequent}$ to an additional $12 \mathrm{~h}$ incubation at $37^{\circ} \mathrm{C}$, the cells were sequentially treated with $3 \% \mathrm{H}_{2} \mathrm{O}_{2}$, blocking buffer (provided in the kit), incubated overnight at $37^{\circ} \mathrm{C}$ with the primary antibody (1:100; anti-cytochrome $c$; cat. no. KG22230; Nanjing KeyGen Biotech Co., Ltd., Nanjing, China). All sections were stained using the streptavidin-horseradish peroxidase complex method with goat anti-rabbit antibodies (1:1,000; provided in the kit) as the secondary antibody at $37^{\circ} \mathrm{C}$ for $30 \mathrm{~min}$, and then $4^{\circ} \mathrm{C}$ overnight. Brown-yellow granules in the cytoplasm represented a positive staining.

The HT-29 cells incubated with different does of SYD were dyed with rhodamine 123 for $1 \mathrm{~h}$ at room temperature. The primary antibody (1:100; anti-cytochrome $c$; cat. no. KG22230) was incubated for $1 \mathrm{~h}$ and then incubated with goat anti-rabbit secondary antibodies with APC fluorescence (cat. no. A-11034; Invitrogen; Thermo Fisher Scientific, Inc.) at $37^{\circ} \mathrm{C}$ for $30 \mathrm{~min}$. Finally, DAPI (cat. no. 40727ES10; Nanjing KeyGen Biotech Co., Ltd.) was directly nucleated for $30 \mathrm{~min}$ at room temperature. The release of cytochrome $c$ was observed under a light microscope (Olympus CX31-LV320 Olympus (China) Co., Ltd., Shanghai, China) subsequent to color development (x400).

Western blot analysis. Western blot analyses were performed as described previously (13) using the following antibodies: Major histocompatibility complex, Class II, DR Beta 4 (DR4; cat. no., 42533S; 1:1,000), major histocompatibility complex, Class II, DR Beta 5 (DR5; cat. no., 3696S; 1:1,000), Fas cell surface death receptor (Fas; cat. no., 8023S; 1:1,000), CASP8 (cat. no., 4927S; 1:1,000), CASP9 (cat. no., 9502S; 1:1,000), CASP3 (cat.no., 9664S; 1:1,000) poly (ADP-ribose) polymerase(PARP; cat. no., 9532S; 1:1,000), $\beta$-actin (cat. no., 4970S; 1:1,000), TNF receptor associated factor 2 (TRAF2; cat. no., 4724S; 1:1,000), TRAIL (cat. no., 3219S; 1:1,000), TNF- $\alpha$ (cat. no., 34; 1:500), X-linked inhibitor of apoptosis (XIAP; cat. no., 14334S; 1:1,000), BCL2, apoptosis regulator (Bcl-2; cat. no., 15071; 1:1,000), BCL2 like 1 (Bcl-xl; cat. no., 2762S; 1:500), MCL1, BCL2 family apoptosis regulator (Mcl-1; cat. no., 94296S; 1:1,000), BCL2 associated X, apoptosis regulator (Bax; cat. no., 2774S; 1:1,000) and truncated (t)-BH3 interacting domain death agonist (BID; cat. no., 8762S; 1:500). All aforementioned antibodies were purchased from Cell Signaling Technology, Inc., (Danvers, MA, USA). In addition, cellular-FLICE-like inhibitory protein (c-FLIP; cat. no., sc-8346, 1:500) was purchased from Santa Cruz Biotechnology, Inc., (Dallas, TX Santa Cruz Biotechnology). Total proteins were extracted from cells using lysis buffer containing phenylmethyl sulfonylfluoride (Beyotime Institute of Biotechnology, Haimen, China) at $25^{\circ} \mathrm{C}$ for $30 \mathrm{~min}$ and protein concentration was determined with a BCA Protein Assay kit (Beyotime Institute of Biotechnology). A total of $20 \mu \mathrm{g}$ protein was separated by SDS-PAGE (10\% gel) and transferred onto a polyvinylidene fluoride membrane. Membranes were blocked with 5\% non-fat milk at room temperature for $2 \mathrm{~h}$ and incubated with primary antibodies aforementioned at $4^{\circ} \mathrm{C}$ for $12 \mathrm{~h}$. Anti- $\beta$-actin (cat. no., 4970S; 1:1,000; Cell Signaling Technology, Inc.) was used as a loading control. Membranes were then washed and incubated with horseradish peroxidase-conjugated secondary antibody (cat. nos. A21020 and A21010; 1:8,000; Abbkine Scientific Co., Ltd., Wuhan, China) at room temperature for $2 \mathrm{~h}$. Immunoreactive bands were visualized using a chemiluminescence solution with an enhanced chemiluminescent kit (Beyotime Institute of Biotechnology). Quantity One (v4.6.8; Bio-Rad Laboratories, Inc., Hercules, CA, USA) was used as the software for quantification.

RNA interference. For the transient knockdown of c-FLIP, the HT-29 cells were transfected with 150 pmol Stealth RNAi siRNA directed against c-FLIP (si-c-FLIP) or non-targeting control siRNA (Scr) (both from Invitrogen; Thermo Fisher Scientific, Inc.) using the TransMessenger transfection reagent kit (Qiagen GmbH, Hilden, Germany). Cells were incubated with the transfection complexes for $4 \mathrm{~h}$ under their normal growth conditions. Subsequently, the complexes were removed from the cells, the cells were washed once with PBS, and then $500 \mu \mathrm{l}$ fresh medium containing FBS and antibiotics (1\% penicillin G-streptomycin; cat. no. 15140122; Thermo Fisher Scientific, Inc.) was added to the cells, according to the manufacturer's protocols. c-FLIP, sense, 5'-CGGACTATA GAGTGCTGATGG-3' and antisense 5'-GATTATCAGGCA GATTCCTAG-3'.

Reverse transcription-quantitative polymerase chain reaction (RT-qPCR) analysis. For the measurement of mRNA expression levels in HT-29 cells with SDY treatment, the mRNA 
expression levels of DR3, DR4, DR5, Fas and TNF- $\alpha$ receptor were detected by RT-qPCR following treatment with $0,10,50$, 100,150 or $200 \mu \mathrm{g} / \mathrm{ml} \mathrm{SYD}$ for $24 \mathrm{~h}$ at room temperature. The housekeeper gene GAPDH was used as a control. The RNA of cells was extracted using TRIzol ${ }^{\circledR}$ reagent (cat. no. 15596018; Invitrogen; Thermo Fisher Scientific, Inc.). cDNA templates from HT-29 cell lines were prepared using a TIANScript RT kit (Tiangen Biotech, Co., Ltd., Beijing, China), according to the manufacture's protocol. RT-qPCR cycling was performed in 96-well plates on a LightCycler 480 Real-Time PCR system (Roche Applied Science, Penzberg, Germany). The reaction was performed in a $20 \mu \mathrm{l}$ total volume containing $10 \mu \mathrm{l} \mathrm{SYBR}{ }^{\circledR}$ Select Master mix (cat. no. 4472908; Thermo Fisher Scientific, Inc.), $1 \mu \mathrm{l}$ each primer $(10 \mu \mathrm{M})$ and $2 \mu \mathrm{l}$ template cDNA. The primer sequences used for PCR are presented in Table I. The amplification protocol consisted of an initial denaturation step at $95^{\circ} \mathrm{C}$ for $5 \mathrm{~min}$, followed by two-step PCR for 40 cycles at $95^{\circ} \mathrm{C}$ for $30 \mathrm{sec}$ and $60^{\circ} \mathrm{C}$ for $30 \mathrm{sec}$. The mRNA expression levels of each target were determined based on the cycle threshold $(\mathrm{Cq})$ value for the reference and each target and calculated as $2-\Delta \mathrm{Cq}$ (5). Three independent experiments were performed.

Statistical analysis. Data were analyzed using SPSS software (version 19.0; IBM Corp., Armonk, NY, USA). The data are presented as the mean \pm standard deviation. The data were analyzed using a two-tailed Student's t-test. For multiple comparisons, a one-way analysis of variance was used with Scheffe as the post-hoc test. $\mathrm{P}<0.05$ or $\mathrm{P}<0.001$ was considered to indicate a statistically significant difference. The calibration was assessed using Hosmer-Lemeshow goodness-of-fit test and Pearson's correlation coefficient was used to calculate the coefficient of correlation.

\section{Results}

Quality control of SYD. For the quality control of SYD, a HPLC analysis was applied in the present study. The chromatogram of a reference standard and a typical chromatogram obtained from the analyses of SYD are indicated Fig. 1. The sulforaphane peak $(9.6 \mathrm{~min})$ in the chromatogram of SYD was identified by comparing the retention time with that of its reference compound (sulforaphane). The linearity of the calibration curve for sulforaphane was assessed at six concentration levels ranging from 0.0281 to $1.4060 \mathrm{mg} / \mathrm{ml}$, and triplicate injections were applied for each concentration. A calibration curve was constructed by plotting the integrated chromatographic peak areas (Y) vs. the corresponding concentration of the injected standard solutions $(\mathrm{X})$. A least squares regression analysis was employed and a regression equation $(\mathrm{Y}=2,623,732.13 \mathrm{X}+7,853.33)$, and coefficient of correlation ( $\mathrm{r}^{2}$ was 0.99990$)$ were obtained over relatively wide concentration ranges for all the analyses. The sulforaphane concentration in the sample was indicated to equal $0.0624 \mathrm{mg} / \mathrm{ml}$.

Effects of SYD on colon cancer cell viability. The antiproliferative effect of SYD in human colorectal cancer cells was tested and it was revealed that SYD inhibited the viability of colon cancer cells in a dose- and time-dependent manner. No significant difference was revealed at the $24 \mathrm{~h}$ time point, but significant differences were noted between $0,10,50,100,200$ and $400 \mu \mathrm{g} / \mathrm{ml} \mathrm{SYD}$ following 48 and $72 \mathrm{~h}$ of treatment $(\mathrm{P}=0.001)$. The viability of HT-29 and LS-174-T cells decreased with increasing doses of SYD at 48 and $72 \mathrm{~h}$, respectively (Fig. 2A and B). At a concentration of $400 \mu \mathrm{g} / \mathrm{ml}$, SYD inhibited the viability of HT-29 and LS-174-T cells. A concentration of $400 \mu \mathrm{g} / \mathrm{ml}$ SYD yielded $\sim 88 / 80$ and $95 / 92 \%$ inhibition of HT-29/LS-174-T cell viability compared with the control group (no treatment) at 48 and $72 \mathrm{~h}$, respectively, and the corresponding half maximal inhibitory concentration $\left(\mathrm{IC}_{50}\right)$ values were $103.89 / 132.05$ and $94.23 / 93.15 \mu \mathrm{g} / \mathrm{ml}$. After $48 \mathrm{~h}$ of treatment with $0-200 \mu \mathrm{g} / \mathrm{ml} \mathrm{SYD}, \mathrm{LDH}$ increased in a dose-dependent manner in the HT29 cell line (Fig. 2C). As a control, the indicated dose of SYD did not significantly decrease the viability of CRL-1790 (normal human colon epithelial) cells, indicating that SYD exhibits selective cytotoxicity in colon cancer cells (Fig. 2D).

SYD-induced cell cycle changes and decrease of $S$-phase cells in HT-29 cells. The effects of SYD on the cell cycle progression of HT-29 cells are indicated in Fig. 3. SYD treatment resulted in a significant dose-dependent accumulation of cells in the G2-M phase at $24 \mathrm{~h}(\mathrm{P}<0.001$, compared with the control; Fig. 3A and B). The population of G2 cells was increased by $6.2,8.4$ and $18.9 \%$ following treatment with 100, 200 and $400 \mu \mathrm{g} / \mathrm{ml} \mathrm{SYD}$, respectively, compared with the control. However, marked G1 arrest was observed with 200 and $400 \mu \mathrm{g} / \mathrm{ml} \mathrm{SYD} \mathrm{following} 48$ and $72 \mathrm{~h}$ treatment (Fig. 3A, C and D). The greatest differences of 41 and $18 \%$ were obtained at 48 and $72 \mathrm{~h}$, respectively, between the 0 and $400 \mu \mathrm{g} / \mathrm{ml} \mathrm{SYD}$ groups, indicating that SYD decreased the population of cells in the $S$ phase.

SYD triggers dose-dependent, caspase-mediated apoptosis. SYD induced dose-dependent apoptosis of HT-29 and LS-174-T cells. The apoptosis significantly increased in the $10,50,100,150$ and $200 \mu \mathrm{g} / \mathrm{ml}$ SYD groups, compared with the $0 \mu \mathrm{g} / \mathrm{ml} \mathrm{SYD} \mathrm{group}(\mathrm{P}<0.05$; Fig. $4 \mathrm{~A})$. The activation of caspases by SYD treatment was subsequently analyzed. A western blot analysis was performed to verify the rapid caspase activation observed with the FACScan assay. The results revealed an accumulation of cleaved CASP8 and CASP3 expression in addition to an increase in the expression level of cleaved CASP9 $(\mathrm{P}<0.001$, compared with the $0 \mu \mathrm{g} / \mathrm{ml} \mathrm{SYD} \mathrm{group;} \mathrm{Fig.} \mathrm{4B).} \mathrm{SYD-induced} \mathrm{apop-}$ tosis was significantly decreased by inhibition of CASP8 (IETD-FMK) compared with control $(\mathrm{P}<0.05)$, whereas the inhibition of CASP9 (LEHD-FMK) had a minimal effect on SYD-triggered HT-29 cell apoptosis (Fig. 4C). Furthermore, the treatment of HT-29 cells with a pan-caspase inhibitor (Z-VAD-FMK) significantly delayed SYD-induced apoptosis compared with control ( $\mathrm{P}<0.05$; Fig. 4C).

SYD regulates the expression of apoptosis-associated genes in colon cancer cells. To determine which apoptosis pathway serves a role in SYD-induced apoptosis, apoptosis-associated genes were first assessed by western blot analysis, which revealed that DR (DR3, DR4 and DR5), tumor necrosis factor- $\alpha$ receptor and the corresponding Fas exhibited minimal alterations in SYD-treated HT-29 cells (data not 
Table I. Primer sequences used for PCR.

\begin{tabular}{lll}
\hline Gene & \multicolumn{1}{c}{ Forward } & \multicolumn{1}{c}{ Reverse } \\
\hline DR3 & 5'-GAGAAGTCCCTGCACCACGA-3' & 5'-TGGGTTCTTTGAGGCTGCTG-3' \\
DR4 & 5'-GCCCCACAACAAAAGAGGTC-3' & 5'-GGAGGTCATTCCAGTGAG TG-3' \\
DR5 & 5'-GCTGGGCATCTGGACCCTCCTACCT-3' & 5'-CAGTCACTTGGGCATTAACACTT-3' \\
Fas & 5'-CCTGTGAGGAGGACGAAC-3' & 5'-CCTGTGAGGAGGACGAAC-3' \\
TNF- $\alpha$ R & 5'-GTCTTCACCACCATGGAG-3' & 5'-CCACCCTGTTGCTGTAGC-3' \\
GAPDH & 5'-TGACTTGATCCGACACATGG-3' & 5'-TCCCATCCATCCAAAAACAT-3'
\end{tabular}

DR, death receptor; TNF- $\alpha$ R, tumor necrosis factor- $\alpha$ receptor; Fas, Fas cell surface death receptor.

shown). Furthermore, a RT-qPCR analysis revealed that the mRNA levels were unchanged (data not shown). In contrast, as the concentration of SYD increased, the expression levels of TRAIL and the Fas ligand (FasLG) also increased. Importantly, the c-FLIP expression levels significantly decreased in a concentration-dependent manner ( $\mathrm{P}<0.05$ for $10-100 \mu \mathrm{g} / \mathrm{ml}$ SYD and P $<0.001$ for $150-200 \mu \mathrm{g} / \mathrm{ml}$ SYD, compared with $0 \mu \mathrm{g} / \mathrm{ml}$ SYD; Fig. 5A).

The activation of CASP8 by SYD encouraged the focus on nuclear factor $\kappa \beta$ (NF- $\kappa \beta)$-regulated proteins that might regulate CASP8 or DR-mediated apoptosis. c-FLIP modulates CASP8 activity by competing with CASP8 for binding at the death domain, which cleaves pro-CASP8 (14). c-FLIP has two alternatively spliced isoforms, short (c-FLIP-S) and long (c-FLIP-L). Although TRAF2 was activated by different doses of SYD, its intensity was weaker than that of CASP8 in HT-29 cells $(\mathrm{P}<0.05$, compared with the $0 \mu \mathrm{g} / \mathrm{ml}$ SYD group; Fig. 5A). The CASP8-processed N-terminal fragment of c-FLIPL is more efficient than c-FLIPL at recruiting TRAF2 and RIP1 (15), as was confirmed by the results of the present study.

c-FLIP decreases the threshold of SYD-mediated growth inhibition. To verify the potential significance of c-FLIP downregulation in SYD cytotoxicity, c-FLIP siRNA was transfected into HT-29 colon cancer cells, which decreased the c-FLIP protein expression levels compared with Src-transfected cells $(\mathrm{P}<0.05$, compared with si-c-FLIP; Fig. 5B). The viability of HT-29 cells was examined with and without c-FLIP knockdown and it was indicated that the knockdown of c-FLIP in HT-29 cells decreased sensitivity to SYD, increased viability and prolonged survival compared with transfection with control siRNA 0, 100 and $200 \mu \mathrm{g} / \mathrm{ml}(\mathrm{P}<0.05$, compared with cells transfected with control siRNA and treated with $100 \mu \mathrm{g} / \mathrm{ml}$ or $200 \mu \mathrm{g} / \mathrm{ml}$ SYD; Fig. 5C). Comparative proliferation assays of cells with and without c-FLIP knockdown were performed and no significant differences were indicated, suggesting that the observed changes in viability are not an artifact of the altered proliferation rate (Fig. 5C and D).

SYD induced XIAP cleavage, the mitochondrial translocation of Bax and tBid and cytochrome discharge. To further confirm the SYD-induced apoptosis pathway, the expression levels of other NF-i $\beta$-regulated proteins were examined. The results
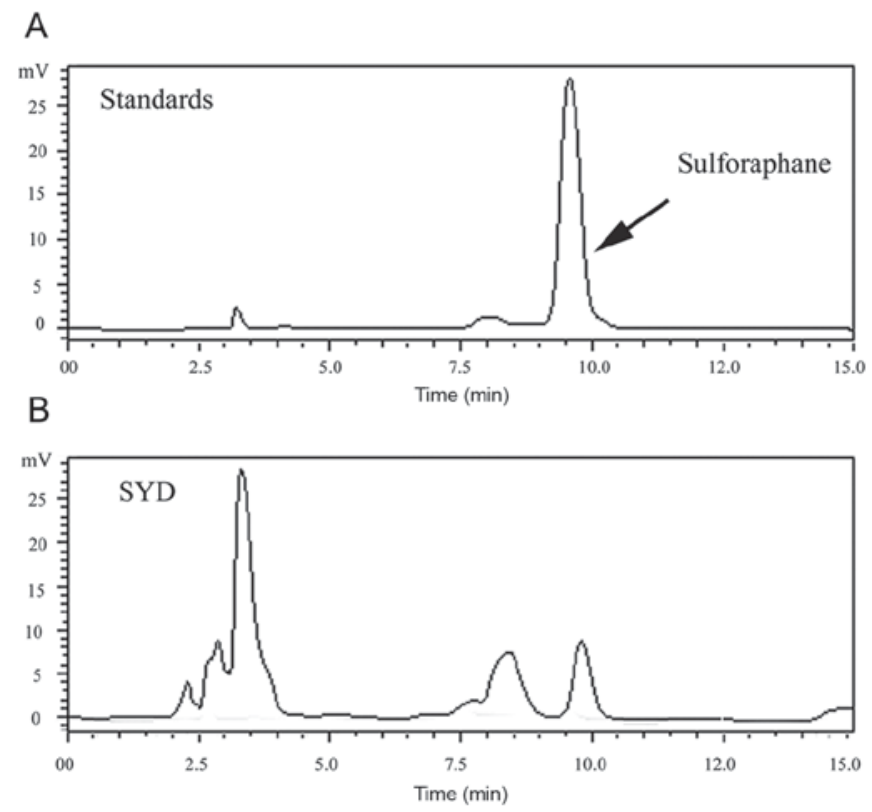

Figure 1. Quality control of SYD. High performance liquid chromatograms of a (A) standard compound (sulforaphane) and a (B) typical sample (SYD). The isocratic elution of methanol and water was set to 20:80 (v/v). The flow rate was $1.0 \mathrm{ml} / \mathrm{min}$, and the detection wavelength was $225 \mathrm{~nm}$. SYD, Su Yang Decoction.

indicated that SYD did not affect the total expression level of the anti-apoptotic Bcl-2 family members, including Bcl-2, Bcl-xl, Mcl-1 and Bax (data not shown). XIAP was indicated to serve as a potent inhibitor of the downstream effectors CASP3, caspase-7 and CASP9, and XIAP cleavage increased in a concentration-dependent manner. The Pan-caspase inhibitor Z-VAD-FMK partially blocked XIAP cleavage, indicating that the inhibition of viability by SYD in colon cancer is partly due to caspase-dependent apoptosis induction $(\mathrm{P}<0.05$, compared with the 0,50 or $100 \mu \mathrm{g} / \mathrm{ml}$ SYD groups; Fig. 6A).

Although the total expression level of pro-apoptotic Bax was not altered, the cytosolic expression level of Bax was decreased and the mitochondrial levels were significantly increased following treatment with increasing concentrations of SYD $(\mathrm{P}<0.05)$. Inversely, increasing concentrations of SYD resulted in significant increases in the levels of cytochrome $c$ in the cytosol and therefore significant decreases in the mitochondrial levels, as determined by western 

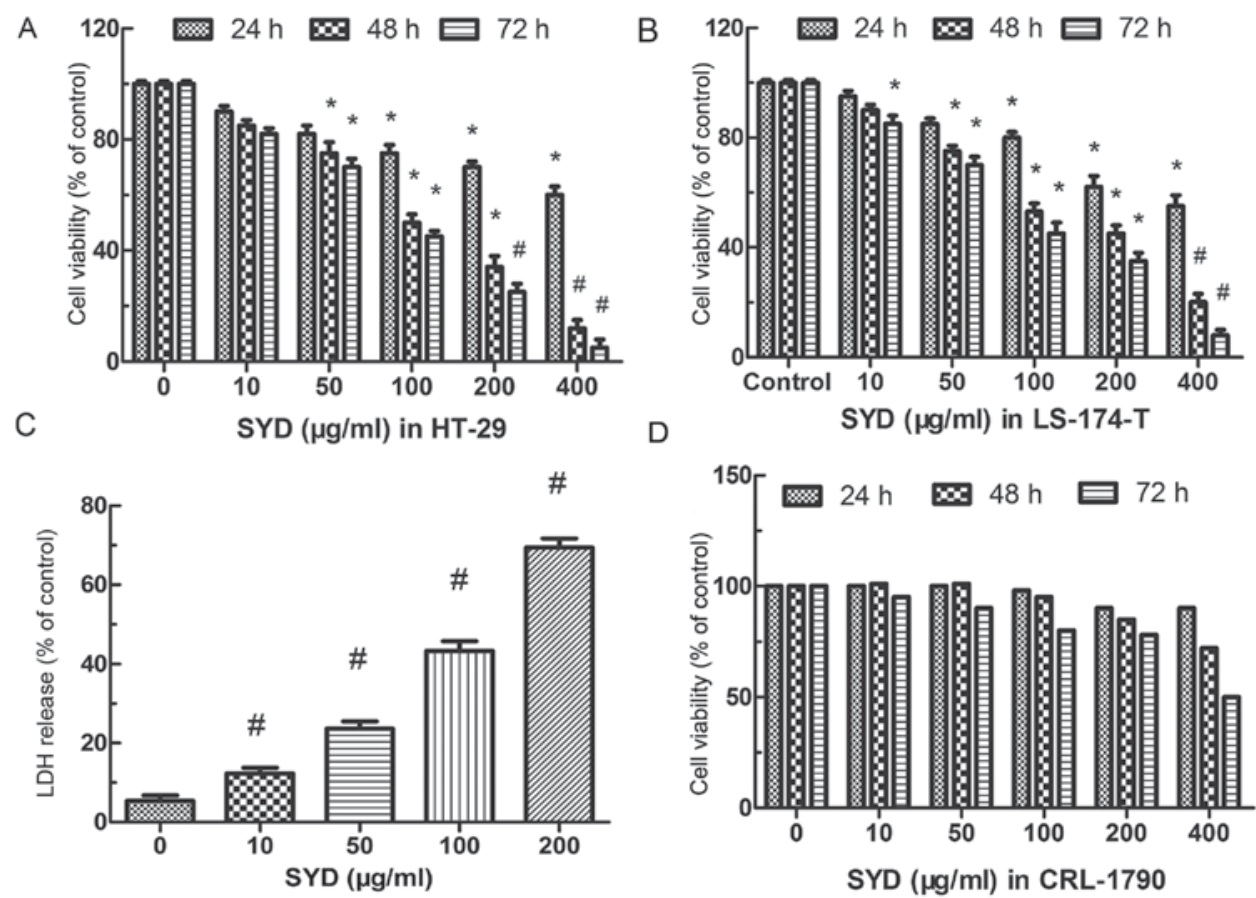

Figure 2. Effects of SYD on colon cancer cell proliferation. SYD affected the proliferation of (A) HT-29 and (B) LS-174-T cells. Adherent cells were plated in 96-well plates and incubated with different concentrations of SYD for 24, 48 and $72 \mathrm{~h}$. (C) Total LDH of HT-29 treated with different dose of SYD for 48 h. The LDH levels in supernatant were examined by an LDH Cytotoxicity Assay kit, according to the manufacturer's protocols. ${ }^{*} \mathrm{P}<0.05$ and ${ }^{\#} \mathrm{P}<0.001$ compared with $0 \mu \mathrm{g} / \mathrm{ml}$ SYD (control). (D) SYD did not affect CRL-1790 cells.
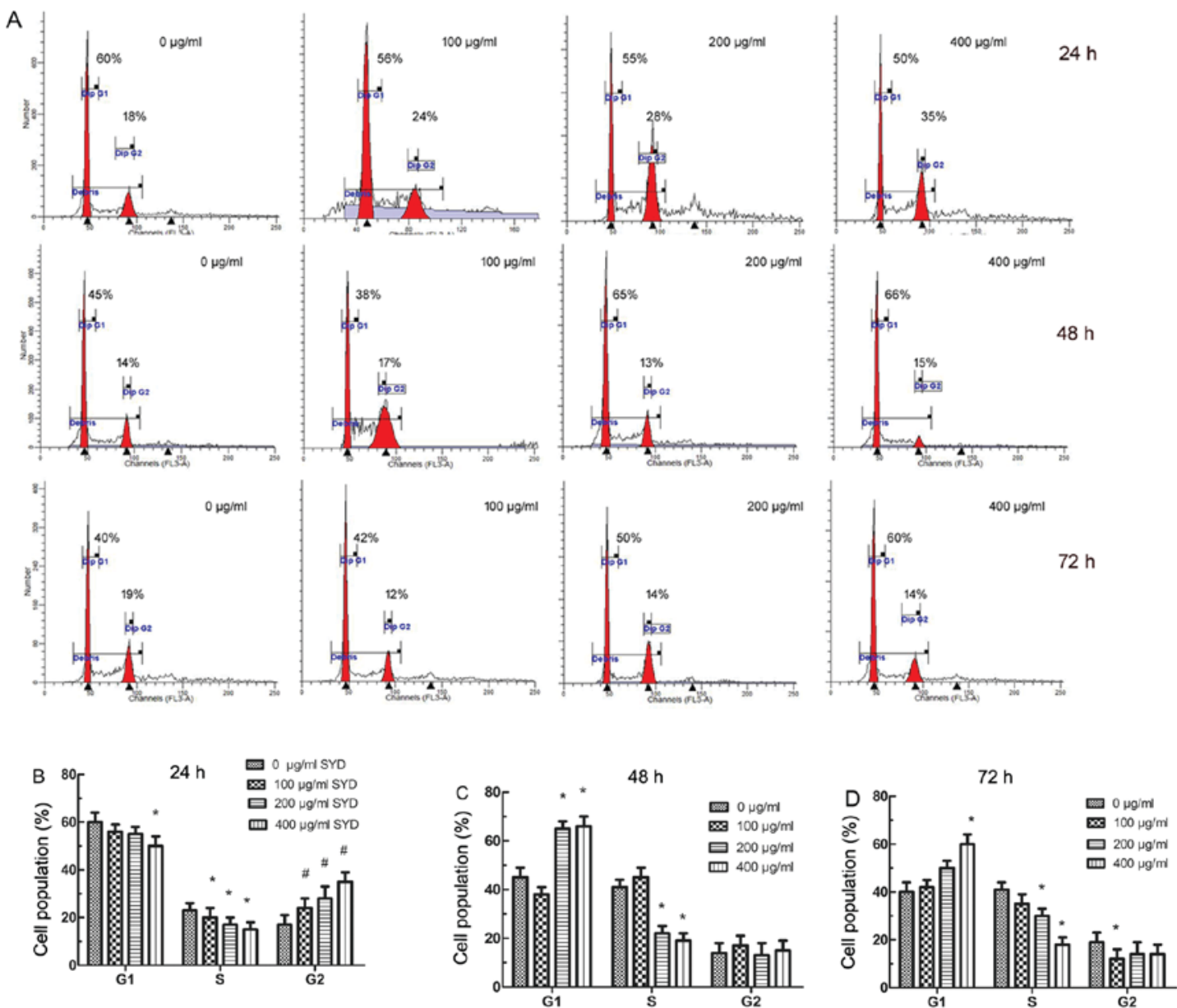

Figure 3. SYD induces cell cycle arrest in human colorectal cancer HT-29 cells. (A) The cells were treated with varying concentrations of SYD (100-200 $\mu \mathrm{g} / \mathrm{ml}$ ) for 24, 48 and $72 \mathrm{~h}$. Quantitative cell cycle distribution data for HT-29 cells following (B) HT-29, (C) LS-174-T and (D) CRL-1790 of treatment. * P<0.05 and ${ }^{\#} \mathrm{P}<0.001$ compared with the control. SYD, Su Yang Decoction. 

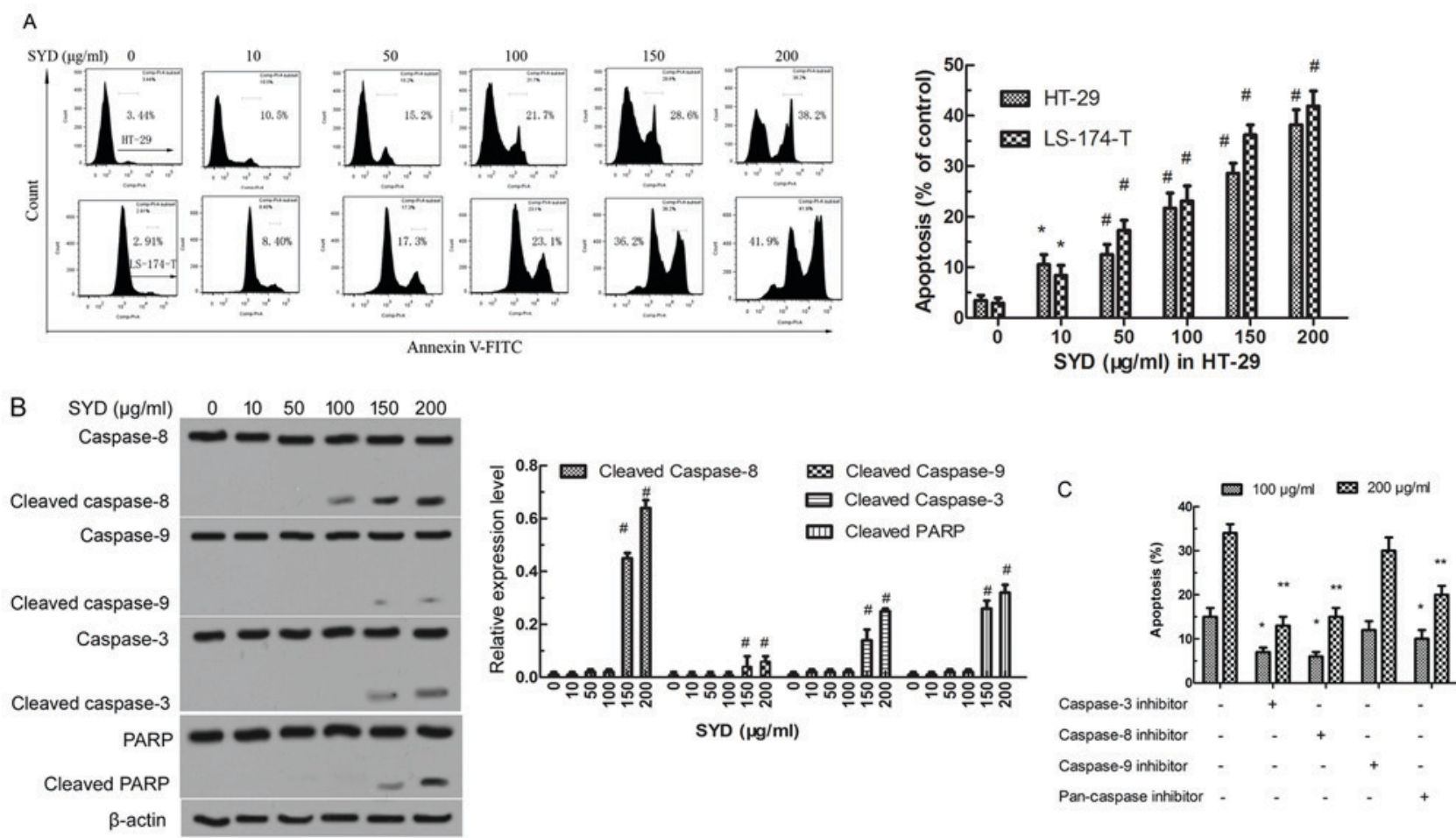

Figure 4. SYD induces apoptosis in a dose-dependent manner. (A) HT-29 and LS-174-T cells were treated with various concentrations of SYD $(0,10,50,100$, 150 , and $200 \mu \mathrm{g} / \mathrm{ml}$ ) for $24 \mathrm{~h}$. ${ }^{*} \mathrm{P}<0.05$ and ${ }^{*} \mathrm{P}<0.001$ compared with $0 \mu \mathrm{g} / \mathrm{ml}$. (B) SYD activates caspases as HT-29 cells were treated with different doses of SYD for $24 \mathrm{~h}$ and incubated with either Caspase-8, Caspase-3, Caspase-9 or PARP antibodies. ${ }^{*} \mathrm{P}<0.001$ compared with $0 \mu \mathrm{g} / \mathrm{ml}$ SYD (control). (C) HT-29 cells were treated with $100 \mu \mathrm{g} / \mathrm{ml}$ or $200 \mu \mathrm{g} / \mathrm{ml} \mathrm{SYD}$ for $24 \mathrm{~h}$ in the presence or absence of biochemical inhibitors of Caspase-3, Caspase-8, Caspase-9 and Pan-caspase. ${ }^{*} \mathrm{P}<0.05$ and ${ }^{* *} \mathrm{P}<0.05$ compared with control group (HT-29 cells with no inhibitor treatment). PARP, poly (ADP-ribose) polymerase; SYD, Su Yang Decoction.

blotting and a cytochrome $c$ assay $(\mathrm{P}<0.05$, compared with the $0 \mu \mathrm{g} / \mathrm{ml}$ SYD group; Fig. 6B and C). Cytochrome $c$ increased with higher doses of SYD (Fig. 6D). Subsequently, a concentration-dependent accumulation of tBid was further revealed. (Fig. 6A). Z-VAD-FMK had no effect on Bid cleavage, indicating a potential caspase-independent underlying mechanism.

\section{Discussion}

Natural products, used as a complementary therapy, have been reported to serve an important role in the treatment of patients with advanced cancer (6). SYD was developed by Infinitus Company Ltd. for the prevention and treatment of colon cancer. SYD has been indicated to improve quality of life (data not shown). However, its multiple constituent compounds and the molecular mechanisms underlying its anticancer activity require further investigation.

SYD is similar in composition to other prescriptions of Chinese herbal compounds. For example, Songyou Yin has been reported to inhibit tumor growth and prolong survival in nude mice bearing a human hepatocellular carcinoma xenograft with high metastatic potential $(16,17)$, however its composition is unknown. It has been reported that SYD comprises two cruciferous vegetable species with some bioactive constituents that exhibit antitumor properties $(4,18)$. It has been demonstrated that sulforaphane, allyl isothiocyanate and phenethyl isothiocyanate in broccoli prevent liver cancer, lung cancer, prostate cancer, breast cancer, rectal cancer and stomach cancer (7). However, it has been indicated that sulforaphane is the best constituent to inhibit the aforementioned cancer types and therefore, sulforaphane constitutes the optimal choice for quality control (8). The present experiment was repeated and it was indicated that the peak at $9.6 \mathrm{~min}$ in the SYD sample corresponds with that in the standard sample. Mass spectrum data further demonstrated that molecular weight 177.2 from sulforaphane standards was in accordance with that in SYD (data not shown), which was hydrolyzed by glucoraphanin in SYD.

Previous epidemiological evidence has indicated that a diet high in cruciferous vegetables may reduce the risk of breast cancer in females (19). The aforementioned data are further supported by data from experimental rodent models treated with the main bioactive components of cruciferous vegetables (data not shown). It has been demonstrated that cell cycle arrest is induced by sulforaphane, which promotes breast cancer cell apoptosis in vitro (20). Sulforaphane has also been indicated to inhibit chemically induced breast cancer in female rats (21), suggesting that sulforaphane may have anticancer activities. Although there are many unknown constituents in SYD, sulforaphane, as the key ingredient in SYD, was tested by HPLC for the quality control of SYD.

The main objective of the present study was to evaluate the anticancer efficacy and associated underlying mechanisms of SYD in human colorectal cancer cells in vitro. It was indicated that SYD inhibited the viability and colony formation of HT-29 and LS-174-T cells. Furthermore, the aforementioned result was also demonstrated by an LDH assay in SYD-treated HT-29 cells (Fig. 2D). In contrast, normal CRL-1790 cells were minimally affected by SYD, even at concentrations that were highly toxic to colon cancer cells. The aforementioned data highlight 

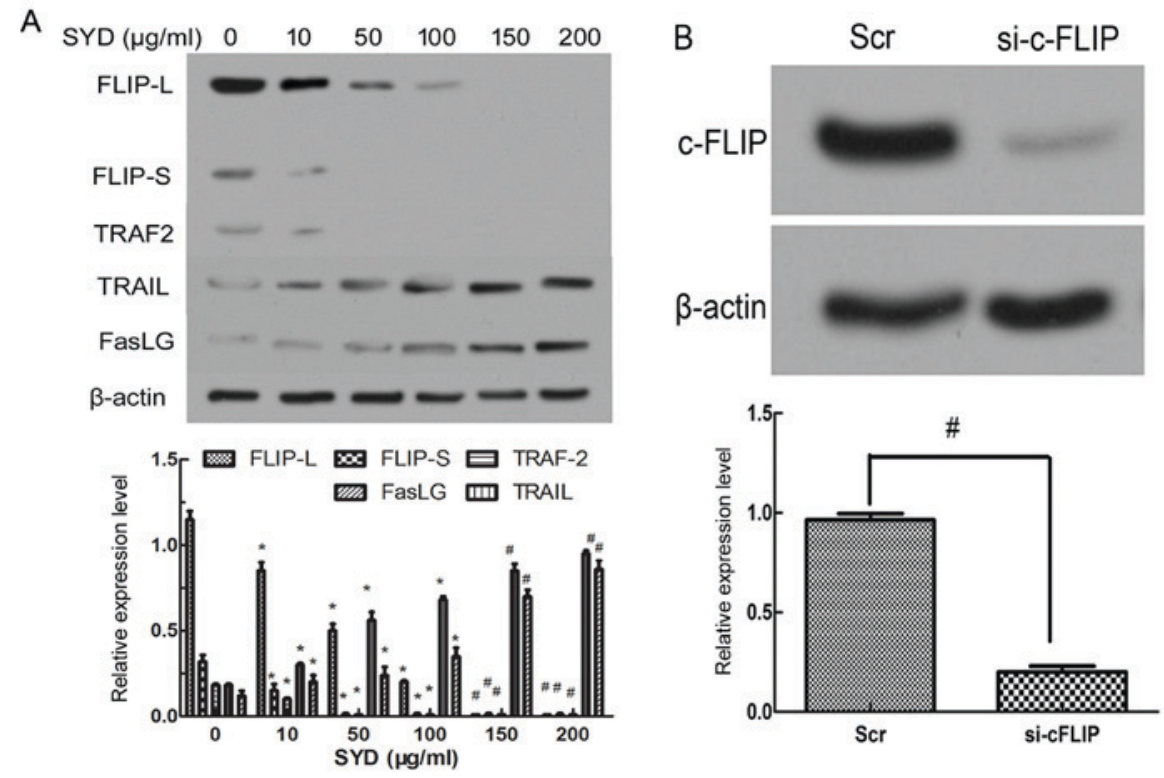

C

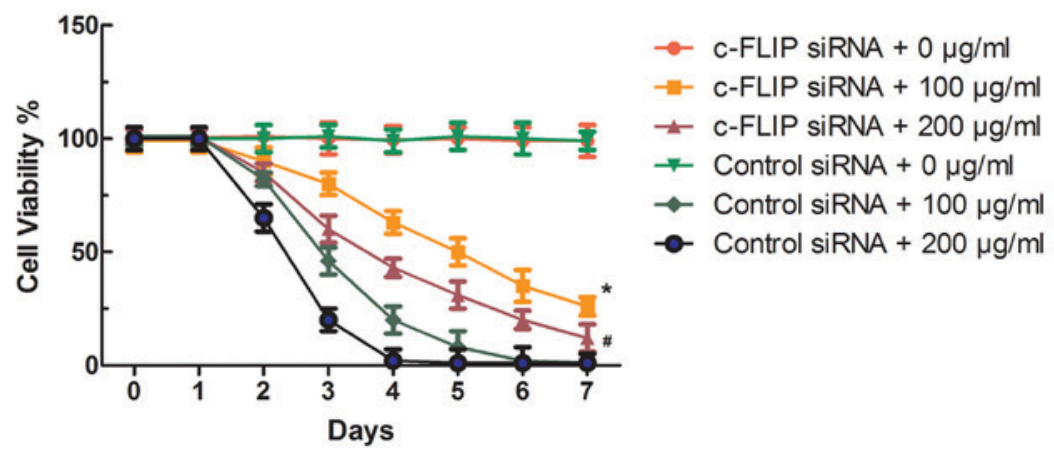

Figure 5. c-FLIP knockout decreases the threshold. (A) Expression levels of FLIP-L, FLIP-S, TRAF2, FasLG, and TRAIL in HT-29 cells treated with different concentrations of SYD for $48 \mathrm{~h}$. "P $<0.05$ and ${ }^{~} \mathrm{P}<0.001$ compared with $0 \mu \mathrm{g} / \mathrm{ml} \mathrm{SYD}$. (B) Expression level of c-FLIP in HT-29 cells transfected with either control siRNA or siRNA targeting c-FLIP. $\beta$-Actin served as the loading control. "P<0.05 for Scr compared with si-c-FLIP. (C) The HT-29 cells were seeded in the presence of SYD $(100 \mu \mathrm{g} / \mathrm{ml}$ or $200 \mu \mathrm{g} / \mathrm{ml})$, and the viability was monitored for 7 days based on the cell count obtained using a hemocytometer with trypan blue staining. The results are expressed as the ratio of viable cells to the total number of cells. "P<0.05 and ${ }^{\#} \mathrm{P}<0.001$ compared with cells transfected with control siRNA and treated with $100 \mu \mathrm{g} / \mathrm{ml}$ or $200 \mu \mathrm{g} / \mathrm{ml} \mathrm{SYD}$, cells transfected with c-FLIP siRNA and not treated or cells transfected with control siRNA and not treated. TRAIL, TNF superfamily member 10; FasLG, Fas ligand; TRAF2, TNF receptor associated factor 2; SYD, Su Yang Decoction; FLICE, FADD-like interleukin-1 $\beta$-converting enzyme; FLIP, FLICE-like inhibitory protein; FLIP-L, long-FLIP; FLIP-S, short FLIP; si/siRNA, small interfering RNA; Scr, scrambled oligonucleotide; c-, cellular.

the importance of broccoli and green cabbage consumption, as natural products, for the prevention and treatment of cancer. In subsequent experiments, SYD was reported to inhibit cell proliferation by inducing cell cycle arrest, specifically in the $\mathrm{S}$ phase, and apoptosis in human colorectal cancer cells in vitro.

Mechanistic experiments from the present study indicated that the anti-colon cancer activity of SYD was associated with the activation of CSPs and c-FLIP. Notably, SYD has been reported to trigger minimal CASP9 cleavage, however robust CASP8 cleavage was observed. The aforementioned data are consistent with the results of the present study, which indicated that biochemical inhibitors of CASP8 had a greater impact on the anti-colon cancer activity of SYD, suggesting that CASP9 serves a minor role in inducing colon cancer apoptosis. Western blot analysis and RT-qPCR assay demonstrated that although the expression levels of DRs, including as DR4, DR5 and Fas, remained unchanged following SYD treatment, SYD considerably increased the levels of their ligands, including TRAIL and FasL (Fig. 5B).
It has been reported that c-FLIP is structurally similar to CASP8 and the expression level of c-FLIP isoforms is increased in various types of cancer, including colon cancer $(22,23)$. Furthermore, it has been demonstrated that the direct siRNA-mediated silencing of c-FLIP increases CASP8 recruitment to the death-enhancing domain and apoptosis induction in some cancer models (24). c-FLIP represents a critical target for therapeutic intervention, which inhibits its transcription and posttranscriptional modification (25). Therefore, the present study suggests that the main toxic effect of SYD results from the downregulation of c-FLIP mediated by SYD. In other words, c-FLIP siRNA decreased the SYD dose needed for apoptosis, suggesting that c-FLIP downregulation is important for SYD cytotoxicity.

Although the expression levels of Bcl-2 and Bax in HT-29 cells were unaffected by SYD, a concentration-dependent increase in the mitochondrial levels of Bax was revealed subsequent to SYD treatment, along with a decrease in the cytoplasmic Bax expression levels. The data of the present 

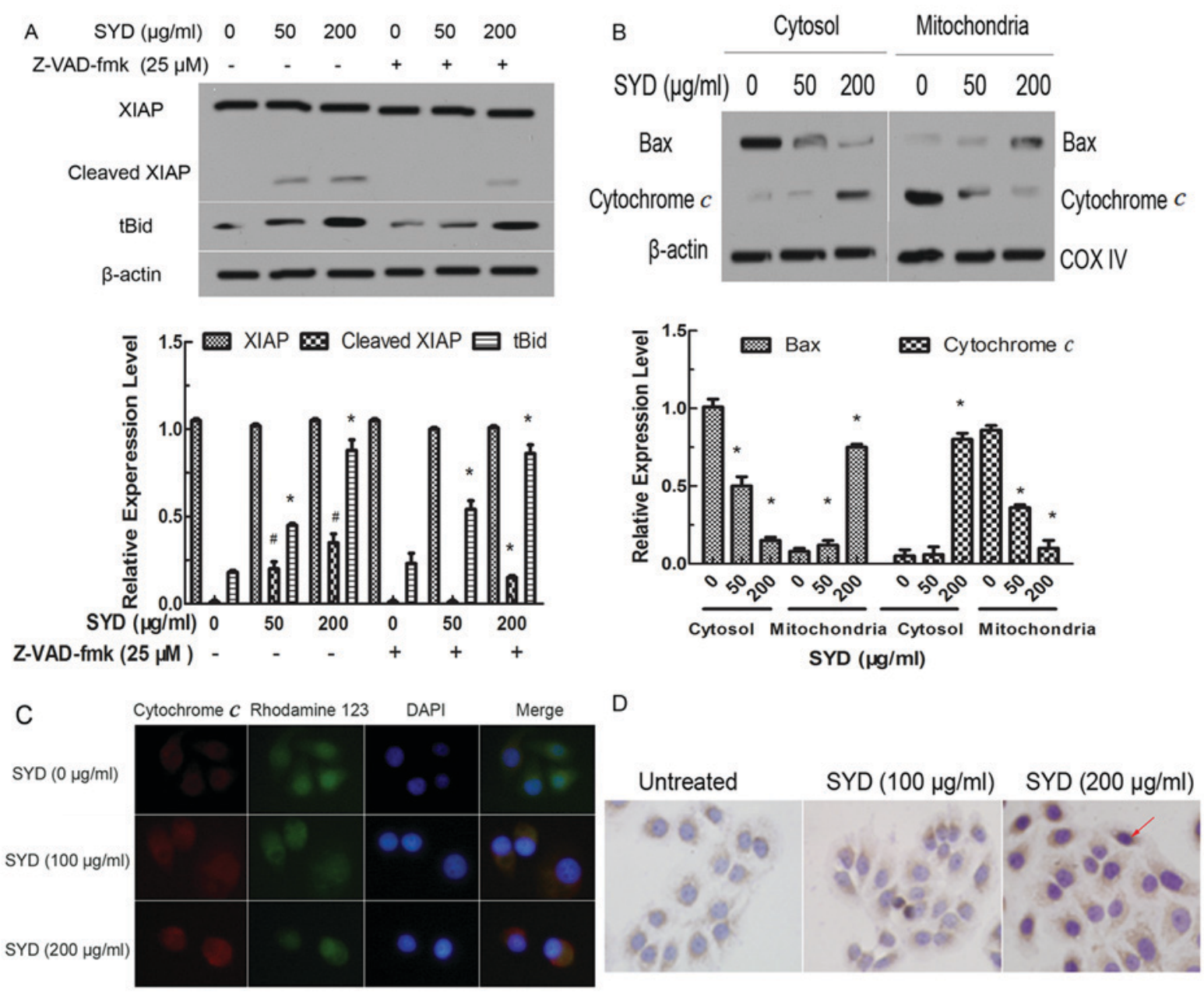

Figure 6. SYD induced XIAP cleavage and the mitochondrial translocation of Bax and truncated Bid. (A) Expression levels of XIAP and tBid in HT-29 cells treated with different doses of SYD with and without Z-VAD-FMK $(25 \mu \mathrm{mol} / \mathrm{l})$ for $24 \mathrm{~h}$. ${ }^{*} \mathrm{P}<0.05$, compared with SYD $(0 \mu \mathrm{g} / \mathrm{ml})$ and ${ }^{\#} \mathrm{P}<0.05 \mathrm{compared}$ with treatment with SYD $(50 \mu \mathrm{g} / \mathrm{ml}$ or $100 \mu \mathrm{g} / \mathrm{ml})$ plus Z-VAD-FMK. (B) Expression levels of the cytosolic and mitochondrial proteins using different doses of SYD treatment $\beta$-Actin and COX IV served as loading controls. "P $<0.05$ compared with $0 \mu \mathrm{g} / \mathrm{ml}$ SYD. (C) Immunofluorescence and (D) immunohistochemical staining of cytochrome $c$ translocated from the mitochondria to the cytosol in HT-29 cells subsequent to incubation with different doses of SYD, including a blank control (magnification, x400). tBid, truncated BH3 interacting domain death agonist; SYD, Su Yang Decoction; COX IV, cytochrome oxidase IV; XIAP, $\mathrm{X}$-linked inhibitor of apoptosis; Bax, BCL2 associated X, apoptosis regulator.

study further demonstrated that SYD treatment yielded a dose-dependent decrease in the release of cytochrome $c$ from the mitochondria accompanied by an increase in the cytosol cytochrome $c$ level. It has been reported that because the release of cytochrome $c$ requires mitochondrial membrane insertion and the oligomerization of Bax, the translocation of Bax proteins from the cytosol to the mitochondria represents a key event in the activation of the intrinsic pathway (25). tBid rapidly binds to membranes and interacts with Bax, causing the insertion of Bax into the membrane and oligomerization, resulting in membrane permeabilization (26). Furthermore, it has been demonstrated that active CASP8 additionally mediates the proteolytic cleavage of Bid to tBid, which is translocated to the mitochondria and amplifies the intrinsic apoptotic pathway (27). Therefore, the data of the present study indicate that SYD induces Bid truncation and Bax translocation to the mitochondria from the cytosol, leading to the release of cytochrome $c$, enhanced mitochondrial dysfunction and the induction of apoptosis in HT-29 cells.

The results of the present study indicated that SYD has anticancer activity in HT-29 and LS-174-T cells and that this formula activates apoptosis and modulates cell cycle regulators by the activation of caspases. Future studies are required to further investigate the exact components of SYD affecting cancer cells in addition to examining the ability of SYD to prevent and treat cancer, the associated underlying mechanism of action and the food-drug interactions of SYD with conventional cancer preventatives and therapeutics. Further testing of control cell lines is required along with in vivo experiments.

\section{Acknowledgements}

The authors would like to thank Dr He Wang and Mr Lei Ma (Cancer treatment center, the Affiliated Second Hospital of Guangzhou Medical University, Guangzhou, China) for their technical assistance.

\section{Funding}

The present study was financially supported by the School Enterprise Cooperation Fund (grant no., 1562005; Guangzhou, China) and the Guangdong Province Medical Science and 
Technology Research Fund (grant no., A2015530; Guangdong, China).

\section{Availability of data and materials}

The datasets used and/or analyzed during the current study are available from the corresponding author on reasonable request.

\section{Authors' contributions}

YZ designed the study and interpreted the results. YG, QG, FY, $\mathrm{XZ}$ and YL collected the test data and drafted the manuscript.

\section{Ethics approval and consent to participate}

The present study was approved by the Institutional Animal Care Committee and the Local Veterinary Office and Ethics Committee of Guangzhou Medical University (Guangzhou, China).

\section{Patient consent for publication}

Not applicable.

\section{Competing interests}

The authors declare that they have no competing interests.

\section{References}

1. Tei M, Otsuka M, Suzuki Y, Kishi K, Tanemura M and Akamatsu H: Safety and feasibility of single-port laparoscopic multivisceral resection for locally advanced left colon cancer. Oncol Lett 15: 10091-10097, 2018.

2. Jin P, Yao D, Xu F, Wang H and Zheng Y: Effect of light on quality and bioactive compounds in postharvest broccoli florets. Food Chem 172: 705-709, 2015.

3. Jiang K, Zhang C, Yu B, Chen B, Liu Z, Hou C, Wang F, Shen H and Chen Z: Autophagic degradation of FOXO3a represses the expression of PUMA to block cell apoptosis in cisplatin-resistant osteosarcoma cells. Am J Cancer Res 7: 1407-1422, 2017.

4. Atay K, Canbakan B, Koroglu E, Hatemi I, Canbakan M, Kepil N, Tuncer M and Senturk H: Apoptosis and disease severity is associated with insulin resistance in non-alcoholic fatty liver disease. Acta Gastroenterol Belg 80: 271-277, 2017.

5. Livak KJ and Schmittgen TD: Analysis of relative gene expression data using real-time quantitative PCR and the 2(-Delta Delta C(T)) method. Methods 25: 402-408, 2001.

6. Wang D, Zhang Y, Lu J, Wang Y, Wang J, Meng Q, Lee RJ, Wang D and Teng L: Cordycepin, a natural antineoplastic agent, induces apoptosis of breast cancer cells via caspase-dependent pathways. Nat Prod Commun 11: 63-68, 2016.

7. Rose P, Huang Q, Ong CN and Whiteman M: Broccoli and watercress suppress matrix metalloproteinase- 9 activity and invasiveness of human MDA-MB-231 breast cancer cells. Toxicol Appl Pharmacol 209: 105-113, 2005.

8. Wang TT, Schoene NW, Milner JA and Kim YS: Broccoli-derived phytochemicals indole-3-carbinol and 3,3'-diindolylmethane exerts concentration-dependent pleiotropic effects on prostate cancer cells: Comparison with other cancer preventive phytochemicals. Mol Carcinog 51: 244-256, 2012.

9. Hensley P, Mishra M and Kyprianou N: Targeting caspases in cancer therapeutics. Biol Chem 394: 831-843, 2013.

10. Liu X, Yue P, Schonthal AH, Khuri FR and Sun SY: Cellular FLICE-inhibitory protein down-regulation contributes to celecoxib-induced apoptosis in human lung cancer cells. Cancer Res 66: 11115-11119, 2006.
11. Mitupatum T, Aree K, Kittisenachai S, Roytrakul S, Puthong S, Kangsadalampai S and Rojpibulstit P: Hep88 mAb-mediated paraptosis-like apoptosis in HepG2 cells via downstream upregulation and activation of caspase-3, caspase-8 and caspase-9. Asian Pac J Cancer Prev 16: 1771-1779, 2015.

12. Fang S, Zhu W, Zhang Y, Shu Y and Liu P: Paeoniflorin modulates multidrug resistance of a human gastric cancer cell line via the inhibition of NF-kappaB activation. Mol Med Rep 5: 351-356, 2012.

13. Wang H, Yin H, Yan F, Sun M, Du L, Peng W, Li Q, Feng Y and Zhou Y: Folate-mediated mitochondrial targeting with doxorubicin-polyrotaxane nanoparticles overcomes multidrug resistance. Oncotarget 6: 2827-2842, 2015.

14. Pop C, Oberst A, Drag M, Van Raam BJ, Riedl SJ, Green DR and Salvesen GS: FLIP(L) induces caspase 8 activity in the absence of interdomain caspase 8 cleavage and alters substrate specificity. Biochem J 433: 447-457, 2011.

15. Dohrman A, Kataoka T, Cuenin S, Russell JQ, Tschopp J and Budd RC: Cellular FLIP (long form) regulates CD8+ T cell activation through caspase-8-dependent NF-kappa B activation. J Immunol 174: 5270-5278, 2005 .

16. Huang XY, Huang ZL, Wang L, Xu YH, Huang XY, Ai KX, Zheng $Q$ and Tang ZY: Herbal compound 'Songyou Yin' reinforced the ability of interferon-alfa to inhibit the enhanced metastatic potential induced by palliative resection of hepatocellular carcinoma in nude mice. BMC Cancer 10: 580, 2010.

17. Zheng S, Jia Q, Shen H, Xu X, Ling J, Jing C and Zhang B: Treatment with the herbal formula Songyou Yin inhibits epithelial-mesenchymal transition in hepatocellular carcinoma through downregulation of TGF- $\beta 1$ expression and inhibition of the SMAD2/3 signaling pathway. Oncol Lett 13: 2309-2315, 2017.

18. Kapiszewska M: A vegetable to meat consumption ratio as a relevant factor determining cancer preventive diet. The Mediterranean versus other European countries. Forum Nutr 59: 130-153, 2006

19. Ambrosone CB, McCann SE, Freudenheim JL, Marshall JR, Zhang Y and Shields PG: Breast cancer risk in premenopausal women is inversely associated with consumption of broccoli, a source of isothiocyanates, but is not modified by GST genotype. J Nutr 134: 1134-1138, 2004.

20. Tseng E, Scott-Ramsay EA and Morris ME: Dietary organic isothiocyanates are cytotoxic in human breast cancer MCF-7 and mammary epithelial MCF-12A cell lines. Exp Biol Med (Maywood) 229: 835-842, 2004.

21. Pajak B, Turowska A, Orzechowski A and Gajkowska B: Bisindolylmaleimide IX facilitates extrinsic and initiates intrinsic apoptosis in TNF-alpha-resistant human colon adenocarcinoma COLO 205 cells. Apoptosis 13: 509-522, 2008.

22. Li TW, Zhang Q, Oh P, Xia M, Chen H, Bemanian S, Lastra N, Circ M, Moyer MP, Mato JM, et al: S-Adenosylmethionine and methylthioadenosine inhibit cellular FLICE inhibitory protein expression and induce apoptosis in colon cancer cells. Mol Pharmacol 76: 192-200, 2009.

23. Sung B, Prasad S, Ravindran J, Yadav VR and Aggarwal BB: Capsazepine, a TRPV1 antagonist, sensitizes colorectal cancer cells to apoptosis by TRAIL through ROS-JNK-CHOP-mediated upregulation of death receptors. Free Radic Biol Med 53: 1977-1987, 2012

24. Moriwaki K, Shinzaki S and Miyoshi E: GDP-mannose-4,6-dehydratase (GMDS) deficiency renders colon cancer cells resistant to tumor necrosis factor-related apoptosis-inducing ligand (TRAIL) receptor- and CD95-mediated apoptosis by inhibiting complex II formation. J Biol Chem 286: 43123-43133, 2011.

25. Kadenbach B, Arnold S, Lee I and Huttemann M: The possible role of cytochrome $\mathrm{c}$ oxidase in stress-induced apoptosis and degenerative diseases. Biochim Biophys Acta 1655: 400-408, 2004.

26. Lovell JF, Billen LP, Bindner S, Shamas-Din A, Fradin C, Leber B and Andrews DW: Membrane binding by tBid initiates an ordered series of events culminating in membrane permeabilization by Bax. Cell 135: 1074-1084, 2008.

27. Xu Z, Tang K, Wang M, Rao Q, Liu B and Wang J: A new caspase- 8 isoform caspase-8s increased sensitivity to apoptosis in Jurkat cells. J Biomed Biotechno 2009: 930462, 2009. 